\title{
September 2015 Pulmonary Case of the Month: Holy Smoke
}

\author{
Samir Sultan, DO \\ David M. Baratz, MD
}

\section{Banner University Medical Center Phoenix \\ Phoenix, AZ}

\section{History of Present IIIness}

A 43-year-old woman presents to the office for second opinion of her dyspnea. She has very mild dyspnea with exertion which she notices when she cannot keep up with people going up stairs. She also has a "smoker's cough".

\section{Past Medical History, Family History, Social History}

Her past medical history, family history and social history are unremarkable other than she smokes 1 ppd for the past 20 years and had a "collapsed lung" about 15 years ago.

\section{Physical Examination}

Her physical examination was unremarkable except for a small scar on her right chest.

\section{Radiography}

A chest $\mathrm{x}$-ray (Figure 1) was performed.

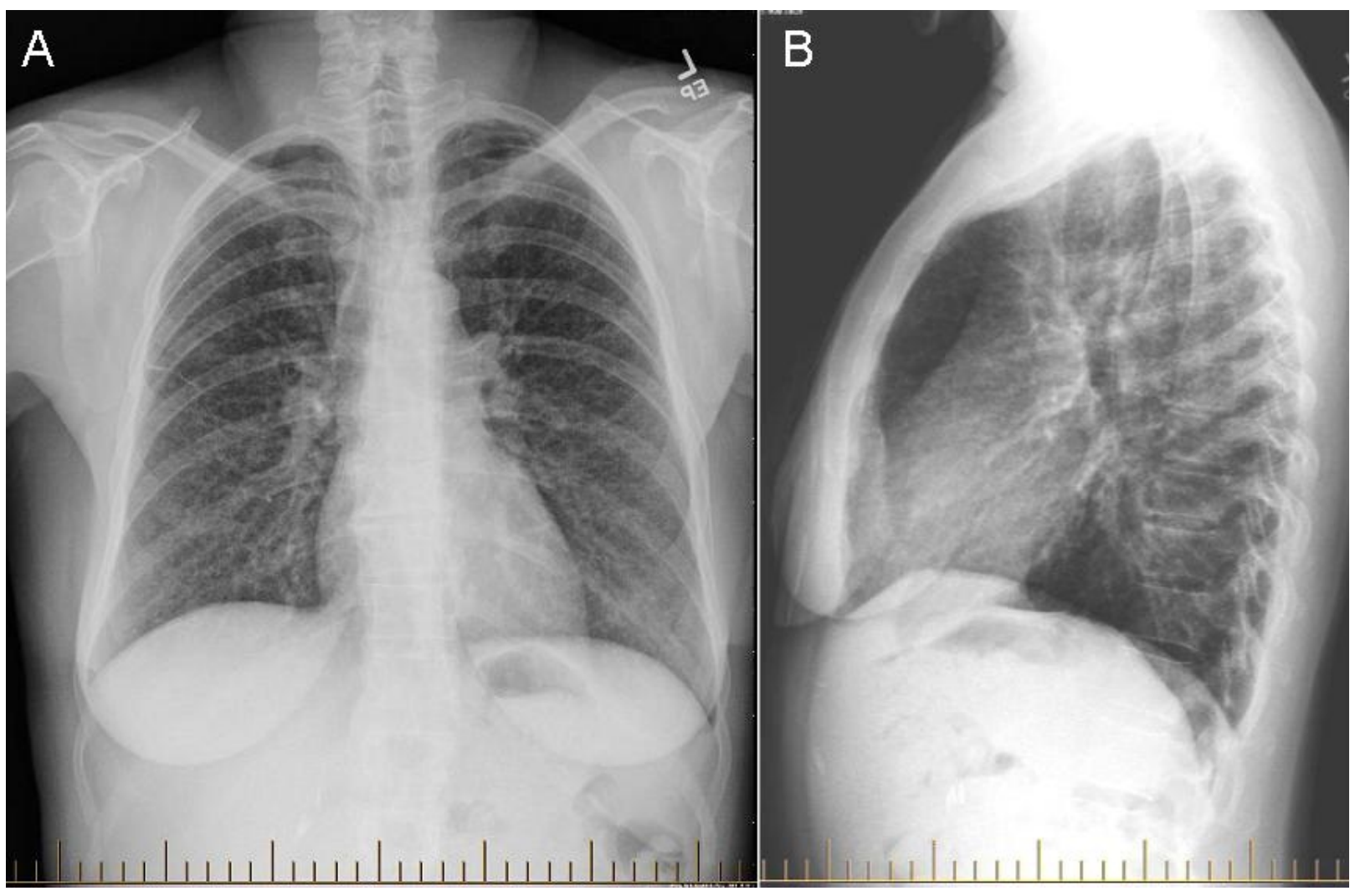

Figure 1. PA (Panel A) and lateral (panel B) chest radiography. 
Which of the following are true regarding the chest x-ray?

1. The chest -ray shows a widened mediastinum

2. The chest $x$-ray is normal

3. The chest $x$-ray shows a diffuse reticulonodular infiltrate

4. The chest $x$-ray shows bilateral hilar adenopathy

5. The chest $x$-ray shows small bilateral pleural effusions 


\section{Correct! \\ 3. The chest x-ray shows a diffuse reticulonodular infiltrate}

Reticular and linear opacification refers to curvilinear opacities that usually involve the lung diffusely. The shadows are caused by thickening of interstitium by blood, water, tumor, cells, and/or fibrous disease. This is best seen in an enlarged view of the right lower lobe (Figure 3).

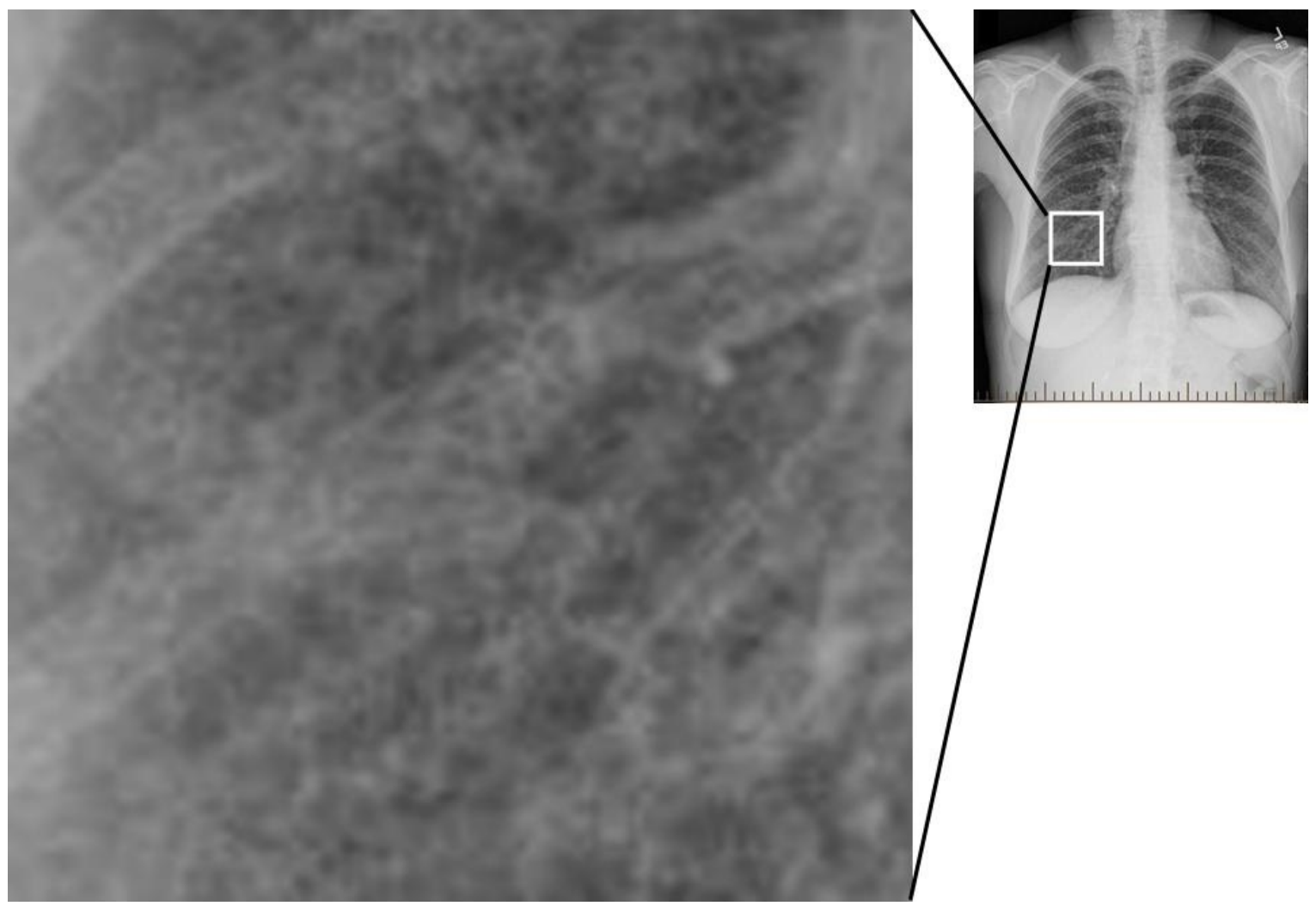

Figure 2. Enlarged area of chest x-ray showing the reticulonodular infiltrates.

There is no evidence of a widened mediastinum, hilar adenopathy or pleural effusions.

Which of the following should not be performed next?

1. Bronchoscopy with transbronchial lung biopsy

2. Pulmonary function testing

3. Thoracic CT scan

4. Ultrasound of the heart

5. All of the above 


\section{Correct! \\ 1. Bronchoscopy with transbronchial biopsy}

Pulmonary function testing revealed a mild-moderate reduction in forced vital capacity (FVC), forced expiratory volume in 1 second (FEV1), total lung capacity (TLC) and diffusing limit for carbon monoxide (DLCo). An ultrasound of the heart specifically looking for evidence of pulmonary hypertension was normal. Thoracic CT scan is shown in Figure 3.

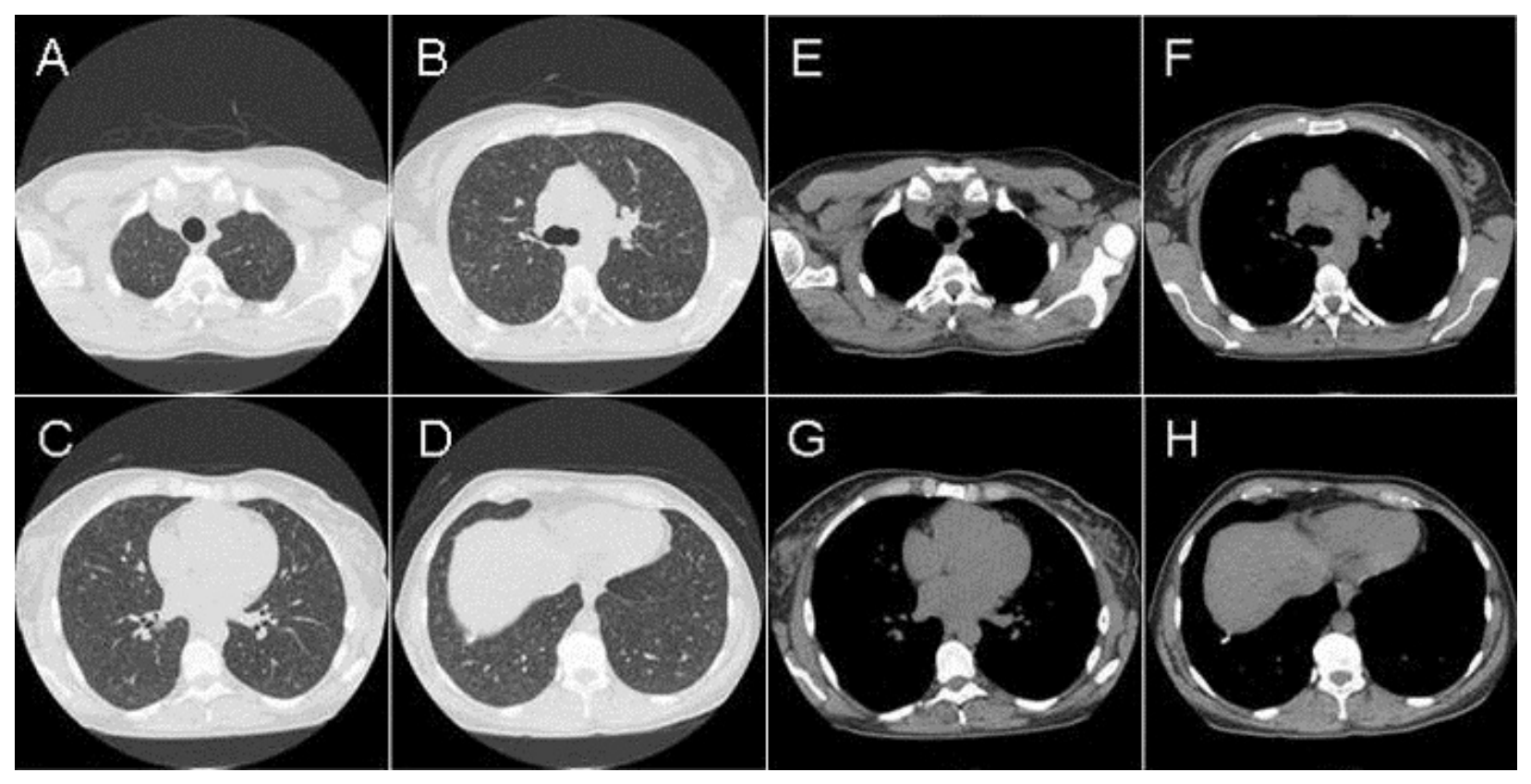

Figure 3. Selected thoracic CT scan in lung windows (panels A-D) and soft tissue windows (Panel E-H).

The CT scan confirms the presence of the reticulonodular infiltrates and also identifies scattered small cysts.

Bronchoscopy with transbronchial biopsy would be reasonable except on further questioning the patient admits to an open lung biopsy about 15 years earlier when she presented with a pneumothorax. The original slides are no longer available but the pathology report was obtained.

Which of the following would you expect on the pathology report?

1. CD1a positive cells

2. Non-necrotizing granulomas

3. S-100 positive cells

4. 1 and 3

5. All of the above 


\section{Correct! \\ 5. All of the above}

The patient was diagnosed with pulmonary Langerhans cell histiocytosis $(\mathrm{PLCH}$, also known by older terminology as eosinophilic granuloma or histiocytosis $X$ ). The classic diagnosis can be made on the basis of compatible imaging showing small nodules and cysts in the upper lungs with bronchoalveolar lavage showing high macrophage counts (1). When patients do not have classic presentation, video-assisted thorascopic surgical (VATS) biopsy is recommended taken from a region with an abundance of nodules. Wedge biopsies of early lesions show multiple well-demarcated grey-white to tan-white irregular, stellate nodules ranging from less than $1 \mathrm{~cm}$ to about $2 \mathrm{~cm}$ (2). With evolution of PLCH, lesions show increasing amounts of stellate fibrosis and cyst formation.

Pathologically, early PLCH lesions consist of discrete bronchiolocentric stellate nodules. Early lesions are more cellular and less fibrotic than more mature lesions and consist of a variable mix of Langerhans cells, lymphocytes, eosinophils, and plasma cells with a background of generally mild fibrosis. Fibrosis replaces the cellular nodules as disease progresses, and less cellular stellate nodules are formed. Langerhans cells usually are immunopositive with CD1a, Langerin, E-cadherin, and S100. Birbeck granules, also termed Langerhans cell granules, pentalaminar rod-shaped cytoplasmic organelles with a racket- or rod-shaped appearance, are found ultrastructurally.

What treatment is most appropriate for this patient with PLCH?

1. Corticosteroids

2. Cytotoxic drugs

3. Lung transplantation

4. Mycophenolate

5. Smoking cessation 


\section{Correct! \\ 5. Smoking cessation}

No randomized trials of therapy for adult PLCH have yet been reported. Data regarding the effectiveness of interventions for PLCH are derived from observational studies, case reports and expert opinion. The majority of PLCH patients are current or former smokers, it is likely that smoking plays a role in the pathogenesis of the disease. Case reports suggest that smoking cessation may lead to marked improvement or resolution of the disease. However, relapse or worsening despite smoking cessation have also been described. Nevertheless, because of the high rate of PLCH-associated pulmonary malignancy, the established relationship between smoking and airflow obstruction and many other health benefits, smoking cessation is the most important step in the management of PLCH and would be most appropriate for this patient with mild disease.

Approximately $50 \%$ of patients have favorable outcomes (1). $10-20 \%$ of patients have recurrent pneumothorax or progressive respiratory failure with cor pulmonale. $30-40 \%$ of patients have persistent symptoms with conversion of the nodules into a more cystic pattern. Poor prognostic indicators include (1):

- Old age

- Constitutional symptoms

- Recurrent pneumothorax

- Extrathoracic lesions (except for bone lesions which has no bearing on prognosis)

- Diffuse cysts on imaging studies

- Severe pulmonary function abnormalities at the time of diagnosis

- Severe pulmonary hypertension

Corticosteroids are frequently used in the management of PLCH despite limited evidence of benefit (3). No trials of treatment with mycophenolate have been reported. Similarly, cytotoxic drugs such as vinblastine, cyclophosphamide, methotrexate, 2chlorodeoxyadenosine (cladribine) and etoposide have been used especially for multisystem disease. Like the corticosteroids there is limited evidence of benefit. Pneumothorax due to PLCH is frequent. Pleurodesis is generally recommended when pneumothorax occurs because the recurrence rate is high. Lung transplantation for $\mathrm{PLCH}$ is performed when severe respiratory failure develops. PLCH is known to recur in the allograft following lung transplantation. None of these therapies seem appropriate for this patient with mild disease.

Our patient is currently attempting to quit smoking. Recidivism to smoking appears to be quite high in $\mathrm{PLCH}$ patients.

\section{References}

1. Tazi A. Adult pulmonary Langerhan's cell histiocytosis. Eur Respir J. 2006;27:127285. [CrossRef] [PubMed] 
2. Allen TC. Pulmonary Langerhans cell histiocytosis and other pulmonary histiocytic diseases: a review. Arch Pathol Lab Med. 2008;132(7):1171-81. [CrossRef] [PubMed]

3. Juvet SC, Hwang D, Downey GP. Rare lung diseases III: pulmonary Langerhans' cell histiocytosis. Can Respir J. 2010;17(3): e55-e62. [PubMed] 